PROCEEDINGS OF THE

AMERICAN MATHEMATICAL SOCIETY

Volume 125, Number 12, December 1997, Pages 3601-3603

S 0002-9939(97)04029-X

\title{
SOME INEQUALITIES DERIVED FROM LOG-SOBOLEV INEQUALITIES
}

\author{
O. S. ROTHAUS
}

(Communicated by Palle E. T. Jorgensen)

\begin{abstract}
We show how a log-Sobolev inequality can be manipulated to give an improved version of itself.
\end{abstract}

There is a very simple manipulation of some log-Sobolev inequalities by means of which the inequality is strengthened, leading in turn to inequalities which could not have been derived from the unimproved version.

We begin with a particularly simple illustration of the manipulation, and will then give a slight generalization to other settings. In order to keep things relatively simple, we will generally work in the smooth (infinitely differentiable) category. Thus we begin with a smooth compact Riemannian manifold, metric scaled so total volume is one, with gradient $\nabla$ and (positive) Laplacian $\Delta$.

Assume we have a defective logarithmic-Sobolev inequality (LSI for short):

$$
\tau \int|\nabla f|^{2} \geq \int f^{2} \ln f^{2}-\int f^{2} \ln \int f^{2}-\rho \int f^{2},
$$

where $f$ is a real smooth function, and $\rho(\rho \geq 0)$ is the defect. As is well known, the above implies

$$
\tau \int|\nabla f|^{2} \geq \int f^{2} V-\int f^{2} \ln \int e^{V}-\rho \int f^{2}
$$

for any smooth $V$, and the modified inequality holding for all such $V$ implies, by a simple approximation argument, the original LSI. The advantage of the modified inequality is its simple quadratic character. In it we may replace $f$ by $f+x$; the left-hand side does not change, so we choose $x$ to maximize the right-hand side. Simple algebra yields the inequality:

$$
\tau \int|\nabla f|^{2} \geq \int f^{2} V-\int f^{2} \ln \int e^{V}-\rho \int f^{2}+\frac{\left(\int f \ln \int e^{V}+\rho \int f-\int f V\right)^{2}}{\ln \int e^{V}+\rho-\int V} .
$$

Since $\rho \geq 0$, the denominator of the last term on the right is positive if $V$ is not constant. If $f$ is not constant, we may now set $V=\ln f^{2}$ (or more properly let $V$ approach $\ln f^{2}$ through a sequence of smooth functions) to get an inequality obviously superior to the original.

Received by the editors July 3, 1996.

1991 Mathematics Subject Classification. Primary 47D07, 58G11.

Key words and phrases. Log-Sobolev inequalities. 
This is not the course we intend to follow. Instead, we suppose $f$ is strictly positive and set

$$
V=\frac{\tau}{2} \frac{|\nabla f|^{2}}{f^{2}}+\frac{\tau}{2} \frac{\Delta f}{f}
$$

This has two consequences; on the one hand $\int V=0$, and on the other, $\tau \int|\nabla f|^{2}=$ $\int_{\text {comes: }} f^{2} V$. With this choice of $V$, set $B=\rho+\ln \int e^{V}$. The modified inequality be-

$$
B \int f^{2} \geq \frac{\left(B \int f-\int f V\right)^{2}}{B}
$$

which we choose to regard as a quadratic inequality for $B$. The inequality

$$
\tau \int|\nabla f|^{2} \geq \int f^{2} V-\int f^{2} \ln \int e^{V}-\rho \int f^{2}
$$

tells us $B$ is non-negative. Thus, solving for the positive root of the corresponding quadratic inequality, yields:

$$
B \geq \frac{\int f V}{\left(\int f^{2}\right)^{1 / 2}+\int f} .
$$

There is a little surprising cancellation taking place here. Rewriting (and using $\left.\int \Delta f=0\right)$ we have

\section{Theorem 1.}

$$
\rho+\ln \int e^{V} \geq \frac{\frac{\tau}{2} \int \frac{|\nabla f|^{2}}{f}}{\left(\int f^{2}\right)^{1 / 2}+\int f}
$$

where $V=\frac{\tau}{2} \frac{|\nabla f|^{2}}{f^{2}}+\frac{\Delta f}{f}$.

Now put $f=e^{u}, u$ arbitrary smooth. The last inequality takes the following form:

Theorem 2.

$$
\rho+\ln \int e^{\frac{\tau}{2} \Delta u} \geq \frac{\frac{\tau}{2} \int e^{u}|\nabla u|^{2}}{\left(\int e^{2 u}\right)^{1 / 2}+\int e^{u}}=\frac{\frac{\tau}{2} \int e^{u} \Delta u}{\left(\int e^{2 u}\right)^{1 / 2}+\int e^{u}} .
$$

This is the inequality alluded to in the introduction. It does not appear to follow directly from the unimproved LSI, nor, for that matter, does it seem to imply the original LSI.

Note that as $\tau$ approaches zero, $\frac{2}{\tau} \ln \int e^{\frac{\tau}{2} \Delta u}$ also approaches zero, while as $\tau \rightarrow \infty$, it approaches max $\Delta u$.

There is a simple variant of our procedure which applies to the original LSI. Here, we suppose we have any smooth Riemannian manifold and a smooth density which gives rise to a modified measure $d m$ of total volume one. Associated to the Dirichlet form $\int|\nabla f|^{2} d m$, we have a perturbed Laplacian $\Delta_{p}$. We work with the algebra generated by compactly supported smooth functions supplemented by constant functions. This algebra is closed under exponentiation. If we have originally a 
defective LSI for this algebra

$$
\tau \int|\nabla f|^{2} d m \geq \int f^{2} \ln f^{2} d m-\int f^{2} d m \ln \int f^{2} d m-\rho \int f^{2} d m,
$$

the same technique used before gives

Theorem 3. For any $u$ in our algebra

$$
\rho+\ln \int e^{\frac{\tau}{2} \Delta_{p} u} \geq \frac{\frac{\tau}{2} \int e^{u}|\nabla u|^{2}}{\left(\int e^{2 u}\right)^{1 / 2}+\int e^{u}}=\frac{\frac{\tau}{2} \int e^{u} \Delta_{p} u}{\left(\int e^{2 u}\right)^{1 / 2}+\int e^{u}},
$$

where all the integrations are with respect to the probability measure $d m$.

Note added in proof. Since this paper was submitted for publication, Prof. M. Ledoux has shown the author a direct proof of an improved version of Theorem 1.

\section{REFERENCES}

1. O. Rothaus, Lower bounds for eigenvalues of regular Sturm Liouville operators and the logarithmic Sobolev inequality, Duke Math. J. 45 (1978), 851-862. MR 58:1368

Department of Mathematics, Cornell University, White Hall, Ithaca, New York 14853-7901

E-mail address: rothaus@math.cornell.edu 